\title{
User Experience Evaluation of Service Platform for Online Courses Based on Fuzzy Comprehensive Evaluation Method-—The User Experience of "Machine Principles" as An Example
}

\author{
Ding Gang ${ }^{1,2}$, Wei Lihong ${ }^{1}$, Cao Danyang ${ }^{2}$, Cheng Yanyu ${ }^{1}$ \\ ${ }^{1}$ Office of Academic Affairs, Harbin Institute of Technology, Harbin City, China \\ ${ }^{2}$ School of Mechatronics Engineering, Harbin Institute of Technology, Harbin City, China

\section{Email address:} \\ dinggang@hit.edu.cn (Ding Gang), weilihong@hit.edu.cn (Wei Lihong), jonacao@yeah.net (Cao Danyang), \\ chyy@hit.edu.cn (Cheng Yanyu)
}

\section{To cite this article:}

Ding Gang, Wei Lihong, Cao Danyang, Cheng Yanyu. User Experience Evaluation of Service Platform for Online Courses Based on Fuzzy Comprehensive Evaluation Method_-The User Experience of "Machine Principles" as An Example. Science Innovation.

Vol. 6, No. 2, 2018, pp. 97-103. doi: 10.11648/j.si.20180602.18

Received: April 17, 2018; Accepted: June 5, 2018; Published: June 22, 2018

\begin{abstract}
The user experience evaluation system of the Service Platform for Online Courses is developed. The platform usability is quantified based on the performance measurement method, and the user experience level of the platform is quantified based on the rank evaluation method. Based on the self-report method, the user experience evaluation of platform has been quantified. On this basis, the user experience evaluation model of the Service Platform for Online Courses based on fuzzy comprehensive evaluation method is developed, and the online course of "machine principle" is taken as an example. The result shows that the overall experience of the Service Platform for Online Course at the present stage is "medium", and the result indicates the direction that the Service Platform for Online Course can continue to improve.
\end{abstract}

Keywords: Fuzzy Comprehensive Evaluation Model, User Experience Evaluation, Service Platform for Online Courses, Machine Principle

\section{基于模糊综合评价法的网络教学平台用户体验测评研究——以 《机械原理》课程用户体验为例}

丁刚 $^{1,2}$, 魏立红 ${ }^{1}$, 曹丹阳 ${ }^{2}$, 程彦宇 ${ }^{1}$

1教务处, 哈尔滨工业大学, 哈尔滨市, 中国

${ }^{2}$ 机电工程学院, 哈尔滨工业大学, 哈尔滨市, 中国

\section{邮箱}

dinggang@hit.edu.cn(丁刚), weilihong@hit.edu.cn(魏立红), jonacao@yeah.net(曹丹阳), chyy@hit.edu.cn(程彦宇)

摘要：本文首先构建了网络教学平台用户体验评价体系。基于绩效度量法对平台可用性进行了量化，基于等级评价法 对平台用户体验等级进行了量化。基于自我报告法对平台用户体验评价进行了量化。在此基础上，构建了基于模糊综 合评价法的网络教学平台用户体验测评模型, 并以《机械原理》在线课程为例进行了验证。测评结果表明：现阶段网 络教学平台总体体验在“中等”范围内, 该结果指出了网络教学平台可以继续提升改进的方向。 
关键词: 模糊综合评价法, 用户体验测评, 网络课程服务平台, 机械原理

\section{1. 引言}

信息化与教学的全面深度融合为教师开展教学改革 提供了宽广舞台 [1-3]。MOOC (Massive Open Online Courses, 大规模开放在线课程) 和SPOC (Small Private Online Course, 小规模限制性在线课程) 以及基于 MOOC/SPOC的“翻转课堂”教学模式很好的体现了信息化 与教学的全面深度融合, 因此在教学实践中得到了广泛应 用[4-6]。为建立“以学生为中心”的教学模式, 作者开发了 基于网络教学平台的《机械原理》在线课程 $[7,8]$ 。

\section{2. 网络教学平台用户体验评价体系的构建}

为评价学生在网络教学平台上进行学习时的用户体验, 走访了 10 名用户体验研究以及交互设计领域的专家, 为确 保专家的专业性, 邀请的专家至少具有 3 年的相关领域从业 经验。在经过三次沟通后得到趋于一致的专家意见, 得到 初步的指标要素分层。并以专家打分法为基础, 基于层次 分析法完成指标提取及权重分配。网络教学平台用户体验 评价指标体系如图1所示, 各级指标权重分配如表1所示。

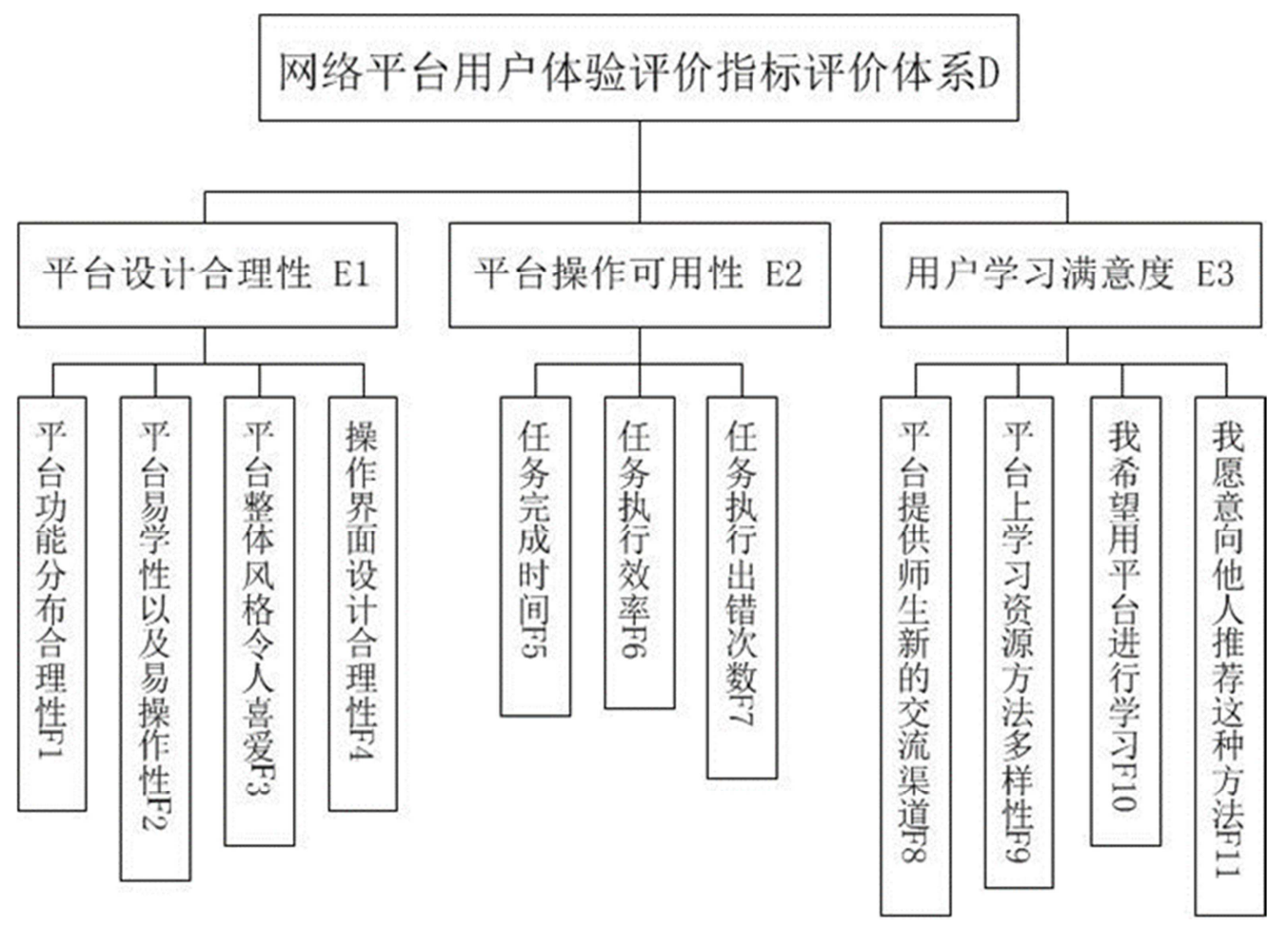

图1 网络教学平台用户体验评价指标体系。

表1 评价体系一级指标和二级指标的权重分配。

\begin{tabular}{llll}
\hline $\begin{array}{c}\text { 一级指标 } \\
\text { Primary index }\end{array}$ & $\begin{array}{l}\text { 权重 } \\
\text { Weight }\end{array}$ & $\begin{array}{l}\text { 二级指标 } \\
\text { Secondary index }\end{array}$ & $\begin{array}{l}\text { 权重 } \\
\text { Weight }\end{array}$ \\
\hline $\boldsymbol{E}_{1}$ & 0.4511 & $\boldsymbol{F}_{1}$ & 0.2456 \\
& & $\boldsymbol{F}_{2}$ & 0.2807 \\
& & $\boldsymbol{F}_{3}$ & 0.2522 \\
& & $\boldsymbol{F}_{4}$ & 0.2215 \\
$\boldsymbol{E}_{2}$ & \multirow{2}{*}{0.3468} & $\boldsymbol{F}_{5}$ & 0.3704 \\
& & $\boldsymbol{F}_{6}$ & 0.2799 \\
& & $\boldsymbol{F}_{7}$ & 0.3496 \\
$\boldsymbol{E}_{3}$ & \multirow{2}{*}{0.2020} & $\boldsymbol{F}_{8}$ & 0.1920 \\
& & $\boldsymbol{F}_{9}$ & 0.2280 \\
& & $\boldsymbol{F}_{10}$ & 0.3293 \\
& & $\boldsymbol{F}_{11}$ & 0.2506 \\
\hline
\end{tabular}

\section{3. 网络教学平台用户体验指标的量化}

\section{1. 基于绩效度量的平台可用性量化分析}

对评价体系中的定量要素, 即平台可用性设计要素进 行度量。采用的方法为绩效度量法, 要求被测人员完成一 系列预先设定好的任务, 从而得出任务的完成时间, 错误 次数, 效率等结果, 所有绩效度量均建立在特定用户行为 的基础上。

此次绩效度量实验为对网络教学平台上的《机械原理》 课程的可用性进行评价。选取 30名年龄在18 30岁之间且 未曾接触过网络教学平台上《机械原理》课程的被测者完 成如表2中所示任务。 
表2 绩效度量任务设置。

\begin{tabular}{ll}
\hline 任务名称 & 任务说明 \\
\hline $\mathrm{T} 0$ & 按要求打开网页http://jwcs.hit.edu.cn/ \\
$\mathrm{T} 1$ & 使用给定账户进行登录 \\
$\mathrm{T} 2$ & 查看全部网络课程 \\
$\mathrm{T} 3$ & 搜索“机械原理”课程 \\
$\mathrm{T} 4$ & 查看课程编号为13SD08000200主讲教师为“丁丁刚”的课程 \\
$\mathrm{T} 5$ & 选修该门机械原理课程 \\
$\mathrm{T} 6$ & 查看齿轮章节的知识架构 \\
$\mathrm{T} 7$ & 学习 (播放) “齿轮机构及其设计”章节中的第一讲 \\
$\mathrm{T} 8$ & 点击进入全屏播放 \\
$\mathrm{T} 9$ & 学习5分钟后点击“暂停”并退出全屏状态 \\
$\mathrm{T} 10$ & 返回课程主菜单 \\
$\mathrm{T} 11$ & 进入“第五章 齿轮机构及其设计”习题 \\
$\mathrm{T} 12$ & 阅读“第五章 齿轮机构及其设计”习题中第16题 \\
$\mathrm{T} 13$ & 观看平台中“机构演示”中第五章第16题 \\
$\mathrm{T} 14$ & 查看课程中“第五章 齿轮机构及其设计”第16题的答案解 \\
T15 & 析 \\
\hline
\end{tabular}

30 名被试均成功的完成了预设任务。被试完成任务的 平均总时间、出错次数以及平均效率如表 3 所示

表3 绩效度量实验结果统计表。

\begin{tabular}{llll}
\hline 项目 & 平均用时 (s) & 平均出错次数(次) & 平均效率 (\%) \\
\hline 数值 & 69.5023 & 2.4194 & 21.58 \\
\hline
\end{tabular}

\section{2. 基于等级评价法的用户体验评价量化}

要求被试在完成操作后对相关项目按照“优秀”、“良 好”、“合格”、“不合格四个”等级进行评价打分，打分等级 与后续的模糊评价等级评语集相对应, 用以后续对定性指 标隶属度的计算。问题设置如表4所示。

表4 用户体验等级评价问卷。

\begin{tabular}{ll}
\hline 问题编号 & 问题内容 \\
\hline Q1 & 平台的功能分布合理 \\
Q2 & 平台的使用容易学习并且操作方便 \\
Q3 & 平台的整体风格令人喜爱 \\
Q4 & 操作界面的设计合理 \\
Q5 & 平台提供给我和老师交流的渠道 \\
Q6 & 我会乐于向他人推荐这种学习方法 \\
Q7 & 平台上的学习资源、学习方法多样 \\
Q8 & 我日后想继续使用平台进行学习 \\
\hline
\end{tabular}

被试打分结果如表5所示。

表5 用户体验等级评价问卷。

\begin{tabular}{lllll}
\hline 问题编号 & 优秀 & 良好 & 合格 & 不合格 \\
\hline Q1 & 4 & 24 & 6 & 0 \\
Q2 & 11 & 18 & 1 & 0 \\
Q3 & 1 & 23 & 6 & 0 \\
Q4 & 5 & 25 & 0 & 0 \\
Q5 & 0 & 15 & 14 & 1 \\
Q6 & 1 & 22 & 7 & 0 \\
Q7 & 12 & 14 & 4 & 0 \\
Q8 & 10 & 18 & 2 & 0 \\
\hline
\end{tabular}

\section{3. 基于自我报告的平台用户体验评价量化}

另以同样标准征集30名被试对评价体系涉及的整体 三方面进行打分评价，即平台设计合理性设计要素、平台 操作可用性以及学习满意度要素进行度量。采用的方法为 自我报告的度量方法, 要求被试在完成平台试用后填写预 先设计好的自我报告调查问卷进而完成此部分的数据采 集。问题设置如表6所示。

表6 自我报告问卷问题。

\begin{tabular}{|c|c|}
\hline 问题编码 & 问题内容 \\
\hline Q1 & 我认为平台的功能整合的很好 \\
\hline Q2 & 我认为平台的操作和功能有很多不一致 \\
\hline Q3 & 我觉得需要有经验的人带我我才能用好平台 \\
\hline Q4 & 平台的整体风格我很喜欢 \\
\hline Q5 & 平台的色彩效果很舒服 \\
\hline Q6 & 我觉着平台用起来有点麻烦 \\
\hline Q7 & 为了能操作好网站, 我要学好多东西 \\
\hline Q8 & 我认为平台很容易使用 ～～～～～ \\
\hline Q9 & 平台上的很多流程不符合我的操作习惯 \\
\hline Q10 & 平台使用逻辑不够连贯 \\
\hline Q11 & 平台的响应速度很快 \\
\hline Q12 & 我觉着大多数人都可以很快上手使用这个平台 \\
\hline Q13 & 平台上的操作反馈很明确 \\
\hline Q14 & 我有时难以理解平台传递给我的信息 \\
\hline Q15 & 平台上的内容设置帮助我更好地理解课程内容 \\
\hline Q16 & 平台能够提供我需要的学习资料 \\
\hline Q17 & 作业中遇到问题可以方便地和老师沟通 \\
\hline Q18 & 平台教学相关的资源比较全面 \\
\hline Q19 & 整体而言, 平台的学习体验令人满意 \\
\hline Q20 & 平台给我提供了愉悦的学习感受 \\
\hline Q21 & $\begin{array}{l}\text { 如果条件允许我愿意尽可能的通过信息化教学平台进行 } \\
\text { 学习 }\end{array}$ \\
\hline Q22 & 我愿意向别人推荐这种学习方法 \\
\hline
\end{tabular}

平台设计合理性打分结果如图2所示。

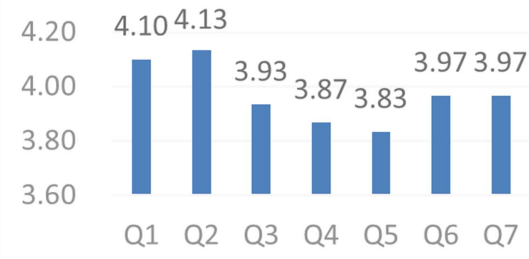

图2 平台设计合理性打分结果。

平台操作可用性打分结果如图3所示。

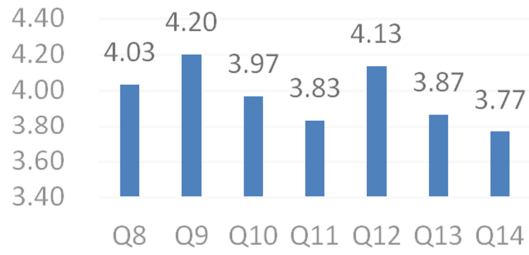

图3 平台操作可用性打分结果。 
网络教学平台学习满意度打分结果如图4所示。

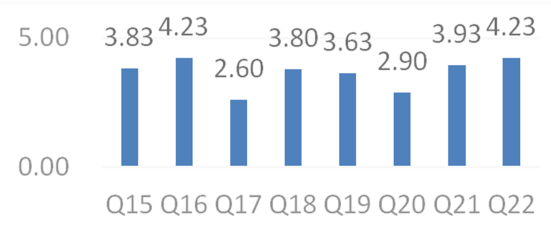

图4 平台学习满意度打分结果。

\section{4. 基于模糊综合评价法的网络教学平台用户体 验测评}

\section{1. 评语集及隶属函数的构建}

网络教学平台用户体验的评语集 $V=\left\{\boldsymbol{v}_{1}, v_{2}, \boldsymbol{v}_{3}, \boldsymbol{v}_{4}\right\}$ 依 据隶属函数建立 [9], 其中 $v_{1}$ 表示体验“优秀”， $v_{2}$ 表示体 验“良好”， $v_{3}$ 表示体验“合格”， $v_{4}$ 表示体验“不合格”。隶 属函数及隶属度的确定方法根据指标的属性不同而有所 差别。定量指标的隶属函数依据被试各指标的操作情况来 进行划分, 根据被试在绩效度量实验中采集的操作数据, 结合评语集的等级内容对其操作表现使用“优秀”“良 好”“合格”“不合格”进行划分。本次平台操作可用性指标下 设三个二级定量指标: 任务完成时间 $\boldsymbol{F}_{5}$ 、任务执行出错 次数 $\boldsymbol{F}_{6}$ 以及任务执行效率 $\boldsymbol{F}_{7}$ 将采用三角形分段函数进行 划分。

对任务的时间指标 $F_{5}$ 分段隶属函数如下: 对于“优秀”的隶属函数, 由式 (1) 求得,

$$
\boldsymbol{V}_{5-1(\boldsymbol{u})}= \begin{cases}1 & 0 \leq \boldsymbol{u}<65 \\ \frac{70-\boldsymbol{u}}{5} & 65 \leq \boldsymbol{u}<70 \\ 0 & \text { 其他 }\end{cases}
$$

对于“良好”的隶属函数, 由式 (2) 求得,

$$
\boldsymbol{V}_{5-2(u)}= \begin{cases}\frac{\boldsymbol{u}-65}{5} & 65 \leq \boldsymbol{u}<70 \\ \frac{75-\boldsymbol{u}}{5} & 70 \leq \boldsymbol{u}<75 \\ 0 & \text { 其他 }\end{cases}
$$

对于“合格”的隶属函数, 由式 (3) 求得,

$$
\boldsymbol{V}_{5-3(\boldsymbol{u})}= \begin{cases}\frac{\boldsymbol{u}-70}{5} & 70 \leq \boldsymbol{u}<75 \\ \frac{80-\boldsymbol{u}}{5} & 75 \leq \boldsymbol{u}<80 \\ 0 & \text { 其他 }\end{cases}
$$

对于“不合格”的隶属函数, 由式 (4) 求得,

$$
\boldsymbol{V}_{5-4(\boldsymbol{u})}= \begin{cases}\frac{\boldsymbol{u}-75}{5} & 75 \leq \boldsymbol{u}<80 \\ 1 & \boldsymbol{u} \geq 80 \\ 0 & \text { 其他 }\end{cases}
$$

通过对被试操作时间的结果进行划分，在65秒内完成 任务全部隶属于“优秀”; 65 70秒完成部分隶属于“优秀”, 部分隶属于“良好”; 70 75秒完成部分隶属于“良好”, 部 分隶属于“合格”; 75 80秒完成部分隶属于“合格”, 部分 隶属于“不合格”; 需要 80 秒以上完成完全隶属于“不合格”。 等级的划分具有模糊性, 即各等级区间间存在着重叠, 隶 属函数将会对重叠部分做出区分, 即明确的指出该结果在 各区间的隶属度大小。

出错次数指标 $\boldsymbol{F}_{6}$ 分段隶属函数如下:

对于“优秀”的隶属函数, 由式 (5) 求得,

$$
\boldsymbol{V}_{6-1(\boldsymbol{u})}= \begin{cases}1 & 0 \leq \boldsymbol{u}<1.5 \\ 2.5-\boldsymbol{u} & 1.5 \leq \boldsymbol{u}<2.5 \\ 0 & \text { 其他 }\end{cases}
$$

对于“良好”的隶属函数, 由式 (6) 求得,

$$
\boldsymbol{V}_{6-2(\boldsymbol{u})}= \begin{cases}\boldsymbol{u}-1.5 & 1.5 \leq \boldsymbol{u}<2.5 \\ 3.5-\boldsymbol{u} & 2.5 \leq \boldsymbol{u}<3.5 \\ 0 & \text { 其他 }\end{cases}
$$

对于“合格”的隶属函数, 由式 (7) 求得,

$$
\boldsymbol{V}_{6-3(\boldsymbol{u})}= \begin{cases}\boldsymbol{u}-2.5 & 2.5 \leq \boldsymbol{u}<3.5 \\ 4.5-\boldsymbol{u} & 3.5 \leq \boldsymbol{u}<4.5 \\ 0 & \text { 其他 }\end{cases}
$$

对于“不合格”的隶属函数, 由式 (8) 求得,

$$
\boldsymbol{V}_{6-4(\boldsymbol{u})}= \begin{cases}\boldsymbol{u}-4.5 & 4.5 \leq \boldsymbol{u}<5.5 \\ 1 & \boldsymbol{u} \geq 5.5 \\ 0 & \text { 其他 }\end{cases}
$$

通过对被试实验期间出错次数的结果进行划分, 完成 任务全过程中错误次数在 1.5 次内全部隶属于“优秀”; 错误 $1.5 \sim 2.5$ 次部分隶属于“优秀”, 部分隶属于“良好”; 错误 2.5 3.5 次部分隶属于“良好”, 部分隶属于“合格”; 错误 3.5 4.5 次部分隶属于“合格”, 部分隶属于“不合格”; 错误 4.5 次以上完成完全隶属于“不合格”。

任务的完成效率 $F_{7}$ 分段隶属函数如下:

对于“优秀”的隶属函数, 由式 (9) 求得,

$$
\boldsymbol{V}_{7-1(\boldsymbol{u})}= \begin{cases}\frac{\boldsymbol{u}-21.5}{1.5} & 21.5 \leq \boldsymbol{u}<23 \\ 1 & \boldsymbol{u} \geq 23 \\ 0 & \text { 其他 }\end{cases}
$$

对于“良好”的隶属函数, 由式 (10) 求得, 


$$
\boldsymbol{V}_{7-2(\boldsymbol{u})}= \begin{cases}\frac{\boldsymbol{u}-20}{1.5} & 20 \leq \boldsymbol{u}<21.5 \\ \frac{23-\boldsymbol{u}}{1.5} & 21.5 \leq \boldsymbol{u}<23 \\ 0 & \text { 其他 }\end{cases}
$$

对于“合格”的隶属函数, 由式 (11) 求得,

$$
\boldsymbol{V}_{7-3(\boldsymbol{u})}= \begin{cases}\frac{\boldsymbol{u}-18.75}{1.25} & 18.75 \leq \boldsymbol{u}<20 \\ \frac{21.5-\boldsymbol{u}}{1.5} & 20 \leq \boldsymbol{u}<21.5 \\ 0 & \text { 其他 }\end{cases}
$$

对于“不合格”的隶属函数, 由式 (12) 求得,

$$
\boldsymbol{V}_{7-4(\boldsymbol{u})}= \begin{cases}1 & 0 \leq \boldsymbol{u}<18.75 \\ \frac{20-\boldsymbol{u}}{1.25} & 18.75 \leq \boldsymbol{u}<20 \\ 0 & \text { 其他 }\end{cases}
$$

通过对被试实验期间完成效率的结果进行划分，完成 任务全过程中平均效率在 $23 \%$ 以上全部隶属于“优秀”; $21.5 \%$ 23\% 部分隶属于“优秀”, 部分隶属于“良好”; $20 \%$ 21.5\% 部分隶属于“良好”, 部分隶属于“合格”; $18.75 \%$ 20\%部分隶属于“合格”, 部分隶属于“不合格”; 效率在0 18.75\%完全隶属于“不合格”。

而定性指标的隶属度则采用等比重法进行确定。由第 四章中被试对于一级指标平台设计合理性 $\boldsymbol{E}_{1}$ 下设的 4 个 二级指标: 平台功能分布合理性 $\boldsymbol{F}_{1}$ 、平台易学性以及易操 作性 $F_{2}$ 、平台整体风格令人喜爱 $\boldsymbol{F}_{3}$ 以及操作界面设计合 理性 $\boldsymbol{F}_{4}$ ；用户学习满意度 $E_{3}$ 下设的 4 个二级指标：平台 提供师生新的交流渠道 $\boldsymbol{F}_{8}$ 、用户愿意向他人推荐这种方 法 $\boldsymbol{F}_{9}$ 、平台上学习资源方法多样性 $\boldsymbol{F}_{10}$ 以及用户希望用平 台进行学习 $\boldsymbol{F}_{11}$ 。给出的“优秀”“良好”“合格”“不合格”的评 价数目, 按等比重进行统计以确定该指标在 4 个评语等级 中的隶属度。

\section{2. 模糊综合评价模型的建立}

模糊综合评价模型的建立依赖于评价指标体系的权 重矩阵 $\boldsymbol{W}_{i}$ 和隶属度形成的单因素评价矩阵 $\boldsymbol{A}_{i}$, 其模型为

$$
\boldsymbol{B}_{i}=A_{i} \bullet W_{i}=\left(b_{i 1}, b_{i 2}, b_{i 3}, b_{i 4}\right)
$$

其运算法则为加权平均

$$
\boldsymbol{b}_{j}=\sum_{k=1}^{m}\left(\boldsymbol{a}_{k}, \boldsymbol{w}_{k j}\right)
$$

其中 $A$ 表示评价指标隶属于 4 个等级隶属度组成的向 量, $W$ 表示该指标占据所属层级的权重, $B$ 为此层级的综 合评判结果。
而为了能够直观准确的将评价隶属于“优秀”“良 好”“合格”“不合格”的程度展示出来, 可以对评分结果应用 式 (15) 进行向百分制的转化。

$$
Q=B^{\prime} \bullet C^{T}
$$

其中, $Q$ 表示综合打分, $B^{\prime}$ 表示评价结果B归一化的 结果, $C^{T}$ 表示四个评语等级对应分数段的中位数。本次 试验中划分分数段为“优秀” 85-100分, “良好”70-85分, “合 格”60-70分, “不合格” $0-60$ 分。其各区间中位数构成的矩 阵 $C^{T}=[92.577 .56530]$ 。

\section{3. 对基于网络教学平台的《机械原理》课程用户体验 进行测评}

由此将之前求得的定性指标和定量指标带入上述模 型计算求得网络教学平台《机械原理》课程的最终得分。 由式 (2) 式 (12) 可以得到二级定性指标的模糊综 合评价结果:

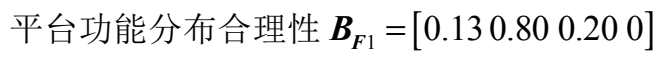

平台易学性以及易操作性 $\boldsymbol{B}_{F 2}=\left[\begin{array}{lll}0.03 & 0.77 & 0.20\end{array}\right]$

平台整体风格令人喜爱 $\boldsymbol{B}_{F 3}=\left[\begin{array}{llll}0.17 & 0.83 & 0 & 0\end{array}\right]$

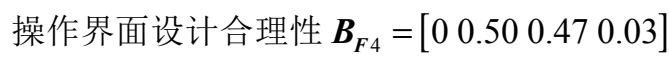

任务完成时间 $\boldsymbol{B}_{F 5}=\left[\begin{array}{llll}0.10 & 0.90 & 0 & 0\end{array}\right]$

任务执行出错次数 $\boldsymbol{B}_{F 6}=\left[\begin{array}{lll}0.08 & 0.92 & 0\end{array}\right]$

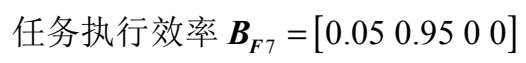

平台提 供师生新的交流渠道 $\boldsymbol{B}_{\boldsymbol{F} 8}=\left[\begin{array}{llll}0.00 & 0.50 & 0.47 & 0.03\end{array}\right]$

用户愿意向他人推荐这种方法 $\boldsymbol{B}_{F 9}=\left[\begin{array}{llll}0.03 & 0.73 & 0.23 & 0\end{array}\right]$

平台上学习资源方法多样性 $\boldsymbol{B}_{F 10}=\left[\begin{array}{llll}0.40 & 0.47 & 0.13 & 0\end{array}\right]$

以及用户希望用平台进行学习 $\boldsymbol{B}_{F 11}=\left[\begin{array}{lll}0.33 & 0.60 & 0.07\end{array}\right]$

依据二级指标隶属度就计算求得一级指标的模糊综 合评价结果为:

平台设计合理性 $\boldsymbol{B}_{E 1}=\left[\begin{array}{llll}0.1810 & 0.7428 & 0.1089 & 0\end{array}\right]$

平台操作可用性 $\boldsymbol{B}_{E 2}=\left[\begin{array}{llll}0.0830 & 0.9169 & 0 & 0\end{array}\right]$

用户学习满意度 $\boldsymbol{B}_{E 3}=\left[\begin{array}{llll}0.2269 & 0.5719 & 0.2047 & 0.0064\end{array}\right]$

依据一级指标隶属度计算求得评价指标的最终的模 糊综合评价结果为:

$$
\boldsymbol{B}_{\boldsymbol{D}}=\left[\begin{array}{lll}
0.1677 & 0.7260 & 0.11430 .0029
\end{array}\right]
$$

将最终评价结果转化为百分制得

$\mathrm{Q}=79.30$ 分

为证明评价体系输出结果合理性, 需对结果的可信度 进行检验。为进行此检验, 本文将模型输出的结果与此前 采取李克特量表法进行打分评价的结果与进行比较, 进而 来分析其合理性。 
由于李克特量表同样可以用来表明被试对某一事物 的态度、看法或评价[10], 结果的得出同样是基于与指标 体系相关的一系列问题, 运用5计量表对答案进行主观向 客观的转化, 再将分值进行加和汇总得到被测群体的主观 态度总分。这个分值越高则体现被试群体对评价对象抱有 的综合态度更积极, 所以其评价效果与模糊评价模型具有 一定的可比性。
本文意在得到被试对于评价对象“网络教学平台《机 械原理》课程”的平均态度, 所以需将被试评价出的量表 总分累加后求得其平均值来得到其平均态度的具体数值。 在对本次李克特量表法问卷中涉及对 22 个与评价指标问 题密切相关的问题进行 $1 \sim 5$ 打分的数据收集后得到表 7 。

表7 李克特量表法结果统计表。

\begin{tabular}{|c|c|c|c|c|c|c|}
\hline $\begin{array}{l}\text { 问题 } \\
\text { 序号 }\end{array}$ & $\begin{array}{l}5 \\
\text { 非常同意 }\end{array}$ & $\begin{array}{l}4 \\
\text { 部分同意 }\end{array}$ & $\begin{array}{l}3 \\
\text { 不一定 }\end{array}$ & $\begin{array}{l}2 \\
\text { 部分反对 }\end{array}$ & $\begin{array}{l}1 \\
\text { 完全反对 }\end{array}$ & 得分均值 \\
\hline Q1 & $30.00 \%$ & $53.33 \%$ & $13.33 \%$ & $3.33 \%$ & $0.00 \%$ & 4.10 \\
\hline Q2 & $33.33 \%$ & $50.00 \%$ & $13.33 \%$ & $3.33 \%$ & $0.00 \%$ & 4.13 \\
\hline Q3 & $30.00 \%$ & $43.33 \%$ & $16.67 \%$ & $10.00 \%$ & $0.00 \%$ & 3.93 \\
\hline Q4 & $20.00 \%$ & $56.67 \%$ & $16.67 \%$ & $3.33 \%$ & $3.33 \%$ & 3.87 \\
\hline Q5 & $26.67 \%$ & $50.00 \%$ & $10.00 \%$ & $6.67 \%$ & $6.67 \%$ & 3.83 \\
\hline Q6 & $26.67 \%$ & $50.00 \%$ & $16.67 \%$ & $6.67 \%$ & $0.00 \%$ & 3.97 \\
\hline Q7 & $36.67 \%$ & $40.00 \%$ & $10.00 \%$ & $10.00 \%$ & $3.33 \%$ & 3.97 \\
\hline Q8 & $30.00 \%$ & $46.67 \%$ & $20.00 \%$ & $3.33 \%$ & $0.00 \%$ & 4.03 \\
\hline Q9 & $33.33 \%$ & $56.67 \%$ & $6.67 \%$ & $3.33 \%$ & $0.00 \%$ & 4.20 \\
\hline Q10 & $23.33 \%$ & $60.00 \%$ & $10.00 \%$ & $3.33 \%$ & $3.33 \%$ & 3.97 \\
\hline Q11 & $26.67 \%$ & $46.67 \%$ & $13.33 \%$ & $10.00 \%$ & $3.33 \%$ & 3.83 \\
\hline Q12 & $23.33 \%$ & $66.67 \%$ & $10.00 \%$ & $0.00 \%$ & $0.00 \%$ & 4.13 \\
\hline Q13 & $30.00 \%$ & $40.00 \%$ & $16.67 \%$ & $13.33 \%$ & $0.00 \%$ & 3.87 \\
\hline Q14 & $16.67 \%$ & $53.33 \%$ & $20.00 \%$ & $10.00 \%$ & $0.00 \%$ & 3.77 \\
\hline Q15 & $30.00 \%$ & $43.33 \%$ & $13.33 \%$ & $6.67 \%$ & $6.67 \%$ & 3.83 \\
\hline Q16 & $36.67 \%$ & $53.33 \%$ & $6.67 \%$ & $3.33 \%$ & $0.00 \%$ & 4.23 \\
\hline Q17 & $6.67 \%$ & $6.67 \%$ & $33.33 \%$ & $46.67 \%$ & $6.67 \%$ & 2.60 \\
\hline Q18 & $13.33 \%$ & $60.00 \%$ & $20.00 \%$ & $6.67 \%$ & $0.00 \%$ & 3.80 \\
\hline Q19 & $6.67 \%$ & $63.33 \%$ & $16.67 \%$ & $13.33 \%$ & $0.00 \%$ & 3.63 \\
\hline Q20 & $6.67 \%$ & $10.00 \%$ & $53.33 \%$ & $26.67 \%$ & $3.33 \%$ & 2.90 \\
\hline Q21 & $20.00 \%$ & $53.33 \%$ & $26.67 \%$ & $0.00 \%$ & $0.00 \%$ & 3.93 \\
\hline Q22 & $40.00 \%$ & $46.67 \%$ & $10.00 \%$ & $3.33 \%$ & $0.00 \%$ & 4.23 \\
\hline 综合评价均分 & & 3.85 & 百分制核算得分 & & 77 & \\
\hline
\end{tabular}

由此可以看出李克特量表得到的对网络教学平台《机 械原理》课程的用户体验主体分布在3 5区间上, 且4分又 占据这其中的大多数, 这与评价模型中对一级指标输出的 评价结果显现出相同的趋势。而对李克特量表的综合评价 均分进行百分制核算后, 求得其结果为77分, 与模糊综合 评价模型输出的79.3分较为接近, 体现了良好的一致性。 由此可以认为模糊综合评价模型给出的评价结果是合理 并且可信的。

经以上模糊综合评价可以看出, 对网络教学平台的 《机械原理》课程用户体验评价打分结果在“中等”范围内, 这证明现阶段其用户体验是大致可以令用户满意的。但通 过实验和模型也暴露出了网络教学平台《机械原理》课程 现阶段存在的不足:

1. 教师与学习者在网络教学平台上的沟通还不能够 畅通, 这种机制的不完善直接导致了用户学习满意度的大 幅下降;

2. 用户在个别操作过程中还存在不顺畅, 逻辑不连 续的情况, 网络教学平台的信息架构还有待调整;

3. 平台上同门课程下设班级过多, 而名称区分度又 较小, 增加了用户进行误操作的可能性, 对用户体验也造 成了一定的影响。

总体来看现阶段网络教学平台仍有较大的提升空间 来获得更好的用户体验, 在日后的完善过程中可以针对本 文研究过程中暴露出的问题进行改进。

\section{5. 结论}

本文构建了网络教学平台用户体验评价体系。为衡量 体系中的定性指标和定量指标, 征集人员使用平台并对问 卷打分, 得到用户对于平台用户体验的主客观评价数据, 通过统计学方法进行处理, 得出被要求评价的各项用户体 验指标的评价结果。最后搭建模糊综合评价模型对网络教 学平台《机械原理》课程用户体验进行评价结果的输出。 模糊评价模型基于实验采集的用户评价数据, 对各个指标 的隶属度进行了划分和计算。最终得到用户对于评价目标 的综合打分。并使用李克特量表转化而来的打分对模型输 出结果进行检验, 衡量其合理性。测评结果表明: 现阶段 网络教学平台总体体验在“中等”范围内, 并依据该结果指 出了平台可以继续提升改进的方向。

\section{致谢}

本文在黑龙江省高等教育教学改革研究重点项目《面 向优质教育资源传播与共享的学分转换机制的研究与实 践》（项目编号：SJGZ20170068）、黑龙江省高等教育 教学改革研究一般项目《MOOC选用的问题分析与对策研 究》 (项目编号: SJGY20170663)、教育部在线教育研 究中心重点项目《基于MOOC的教育资源建设和增益传播 的理论与实践研究》的阶段性成果之一。 


\section{参考文献}

[1] Ding Gang, Zhang Yangyang. Research on MOOC domestic learning user groups [J]. Science Innovation, 2017, 5(1): $38-43$.

[2] 李曼丽. MOOCs的特征及其教学设计原理探析[J]. 清华大 学教育研究,2013,4:13-21。

[3] Jeremy Knox. Digital culture clash: "massive" education in the E-learning and Digital Cultures MOOC[J]. Distance Education, 2014, 35(2): 164-177.

[4] 徐晓飞,傅育熙,李廉,等.关于我国计算机教育MOOC发展的 思考[J].中国大学教学, 2015, 11: 6-10+30。

[5] LNA Atiaja, RSG Proenza. The MOOCs: origin, characterization, principal problems and challenges in Higher

Education [J]. Journal of E-Learning and Knowledge Society, 2016, 12(1): 65-76.

[6] 徐银波,许小芳.发展与困境: “慕课”的现状剖析 [J]. 黑龙江 教育学院学报,2016,3:42-45.

[7] 哈尔滨工业大学.关于本科教学管理与服务平台及网络课 程服务平台投入运行的通知 [EB/OL]. http://today.hit.edu.cn/news/2015/02-27/8621132120RL0.htm, 2018-04-1。

[8] 丁刚,蔡冰倩.面向MOOC的“机械原理”动态教学系统的设 计与开发 $[\mathrm{J}]$.广西大学学报(自然科学版),2015,40(增刊2): 140-144。

[9] 王秦,赵玮.基于模糊综合评价法的我国职业教育供求协调 发展评价指标体系构建 [J].现代教育管理,2018,2: 94-99。

[10] 吴清.基于李克特量表的消费养老方式认知度调查 $[\mathrm{J}]$.技术 与市场, 2013, 8: 188-189。 